\title{
A CHARACTERIZATION OF ARTINIAN RINGS
}

\author{
by DINH VAN HUYNH and NGUYEN V. DUNG
}

(Received 3 May, 1986)

1. Introduction. Throughout this paper we consider associative rings with identity and assume that all modules are unitary. As is well known, cyclic modules play an important role in ring theory. Many nice properties of rings can be characterized by their cyclic modules, even by their simple modules. See, for example, [2], [3], [6], [7], [13], [14], [15], [16], [18], [21]. One of the most important results in this direction is the result of Osofsky [14, Theorem] which says: a ring $R$ is semisimple (i.e. right artinian with zero Jacobson radical) if and only if every cyclic right $R$-module is injective. The other one is due to Vamos [18]: a ring $R$ is right artinian if and only if every cyclic right $R$-module is finitely embedded.

Starting from the Osofsky's result, Boyle has introduced an interesting class of rings whose proper cyclic right modules are injective (the right PCI rings for short). Right PCI rings and related rings have drawn the attention of many authors (see, for example, [3], [4], [7], [9]). One other type of investigation in this direction is to characterize rings by means of their cyclic right modules all of which are assumed to satisfy some decomposition properties (see, for example, [15], [16], [2], [6]). Following the investigation of Smith in [15], Chatters [2, Theorem 3.1] obtained a nice characterization of right noetherian rings as rings whose cyclic right modules are direct sums of a projective module and a noetherian module.

In connection with all that above, we shall prove the following theorem.

THEOREM 1.1. A ring $R$ is right artinian if and only if every cyclic right $R$-module is a direct sum of an injective module and a finitely embedded module.

Using this theorem, we can improve the module characterization of hereditarily artinian rings given in [6, Theorem 1] and prove some related results (Theorem 4.1, Propositions 4.2, 4.3).

2. Preliminaries. Let $R$ be a ring. For a module $M, M_{R}$ means that $M$ is a right $R$-module, $\operatorname{Soc}\left(M_{R}\right)$ denotes the socle of $M_{R},+$ and $\oplus$ stand for a module and ring theoretic direct sum, respectively. A submodule $H$ of a module $M$ is called essential in $M$ if for each non-zero submodule $N$ of $M, H \cap N \neq 0$. A module $M$ is defined to be finitely embedded if $\operatorname{Soc}(M)$ is finitely generated and essential in $M$. Now, we say that a ring $R$ satisfies the property (P) if every cyclic right $R$-module is a direct sum of an injective module and a finitely embedded module.

LeMMA 2.1. If $R$ is a ring satisfying $(P)$ then every homomorphic image of $R$ satisfies (P) too.

Proof. Straightforward.

Glasgow Math. J. 30 (1988) 67-73. 
A ring $R$ is called right self-injective if $R_{R}$ is injective, and $R$ is called regular (in the sense of von Neumann) if $a \in a R a$ for any $a \in R$. The following statement was proved by Osofsky [14, Lemma 5].

Lemma 2.2. Let $R$ be a right self-injective regular ring and $\left\{e_{i}\right\}_{i=1}^{\infty}$ an infinite set of orthogonal idempotents of $R$. Then $R /\left(\sum_{i=1}^{\infty}{ }^{+} e_{i} R\right)$ is not injective over $R$.

LeMma 2.3. Let $R$ be a regular ring and $e$ an idempotent of $R$. Then, for any $a \in R$, there exists an idempotent $f \in R$ such that $e f=f e=0$ and $e R+a R=e R+f R$.

Proof. See Lambek [12, p. 111].

A ring $R$ is called an RM ring if, for each non-zero ideal $I$ of $R, R / I$ is right artinian. As a generalization of the Vamos result mentioned in the introduction, Armendariz and Hummel [1, Proposition 3.1] proved the following result.

LEMMA 2.4. Let $R$ be a ring such that, for each non-zero ideal $I$ of $R, R / I$ is a finitely embedded right $R / I$-module. Then $R$ is an $R M$ ring.

3. Proof of Theorem 1.1. It is clear that every right artinian ring satisfies (P). Assume now that $R$ is a ring satisfying (P). We first consider the case that $R$ is semiprime. By (P) we have

$$
R_{R}=A+B,
$$

where $A_{R}$ is a finitely embedded module and $B_{R}$ is injective. Since $R$ is semiprime, it is easy to see that $A_{R}=\operatorname{Soc}\left(A_{R}\right)$ is a direct sum of finitely many minimal idempotent right ideals of $R$. Without loss of generality, we can assume that $A_{R}$ contains no non-zero injective submodules. Let $C$ be the sum of all non-injective simple submodules of $R_{R}$. Then $C$ is an ideal of $R$, obviously. Since $A \subseteq C$ we get, by (1), $C=A+C \cap B$. Since $\operatorname{Soc}\left(C_{R}\right)=C$, every minimal submodule of $C_{R}$ is non-injective. On the other hand, every minimal submodule of $B_{R}$ is injective by the injectivity of $B_{R}$ and semiprimeness of $R$. Hence $C \cap B=0$, which implies $C=A$. Then the fact that $R$ is semiprime forces $R=A \oplus B$ at once. From this $A$ is a semisimple ring and $B$ is a right self-injective regular ring satisfying $(\mathrm{P})$.

In order to show that $B$ is right artinian, it is enough to show that $B$ does not contain an infinite set of orthogonal idempotents. Assume the contrary that $B$ contains an infinite set $\left\{e_{i}\right\}_{i=1}^{\infty}$ of orthogonal idempotents $e_{i}$. Put

$$
D=\sum_{i=1}^{+} e_{i} B .
$$

Then the cyclic right $B$-module $\bar{B}=B / D$ has a direct decomposition

$$
\bar{B}=\bar{K}+\bar{H},
$$

where $\bar{K}_{B}$ is injective and $\bar{H}_{B}$ is finitely embedded. Let $H$ be the inverse image of $\bar{H}$ in $B$. Then $B / H$ is injective over $B$ and $H / D$ is finitely embedded over $B$. We first show that 
$H_{B}$ is injective. If $B=H$, the statement is clear. We consider now the case $B \neq H$. It follows that

$$
H=a B+\sum_{i=1}^{\infty} e_{i} B
$$

for some $a \in H$. For $a B+e_{1} B$, Lemma 2.3 shows the existence of an idempotent $f_{0}$ of $B$ with $f_{0} f_{1}=f_{1} f_{0}=0$ (here we set $f_{1}=e_{1}$ ) and $a B+e_{1} B=f_{0} B+f_{1} B$. Using Lemma 2.3, we can inductively show that

$$
a B+e_{1} B+\ldots+e_{n} B=f_{0} B+f_{1} B+\ldots+f_{n} B
$$

holds for each $n=1,2, \ldots$, where $\left\{f_{j}\right\}_{j=0}^{n}$ is a system of orthogonal idempotents. Hence

$$
H=\bigcup_{n=1}^{\infty}\left(a B+e_{1} B+\ldots+e_{n} B\right)=\bigcup_{n=0}^{\infty}\left(f_{0} B+\ldots+f_{n} B\right)=\sum_{j=0}^{+} f_{j} B .
$$

Since $B / H$ is injective, Lemma 2.2 allows only finitely many non-zero $f_{j}$ to occur in $\left\{f_{j}\right\}_{j=0}^{\infty}$, say $f_{0}, \ldots, f_{m}$. Let $f=f_{0}+\ldots+f_{m}$. Then $H=f B$; therefore $H_{B}$ is injective.

Since the right $B$-module $H / D$ has a finitely generated essential socle, $H / D$ clearly has finite Goldie dimension, $k$ say. Let $I_{1}, \ldots, I_{k+1}$ be infinite subsets of the index set $\{1,2, \ldots\}$ such that

$$
I_{1} \cup I_{2} \cup \ldots \cup I_{k+1}=\{1,2, \ldots\}
$$

and $I_{i} \cap I_{j}=\varnothing$ for $i \neq j$. Put

$$
S_{i}=\sum_{j_{i} \in I_{i}}^{\dot{+}} e_{j_{i}} B \quad\left(e_{j_{i}} \in\left\{e_{i}\right\}_{i=1}^{\infty}\right)
$$

Then

$$
D=S_{1}+\ldots+S_{k+1}
$$

Let $E\left(S_{i}\right)$ be the injective hull of $S_{i}$ in $H$ with $E\left(S_{i}\right)_{\supseteq} S_{i}$. Then we get

$$
H=E\left(S_{i}\right)+\ldots+E\left(S_{k+1}\right)+H^{\prime} .
$$

By (2) and since $H$ is cyclic, $S_{i} \neq E\left(S_{i}\right)$ for each $i=1, \ldots, k+1$. From this, it is easy to see that the Goldie dimension of $H / D$ is at least $k+1$, a contradiction. Hence $B$ does not contain infinite sets of orthogonal idempotents. It follows that $B$ and therefore $R$ is a semisimple ring.

Now we go to the general case. Let $N$ be the prime radical of $R$. Then $\bar{R}=R / N$ is a semisimple ring by Lemma 2.1 and the consideration above. As is well known, $\bar{R}_{\bar{R}}$ has the direct decomposition

$$
\bar{R}=\bar{e}_{1} \bar{R}+\ldots+\bar{e}_{m} \bar{R}
$$

where each $\bar{e}_{i} \bar{R}$ is a minimal right ideal of $\bar{R}$ and $\left\{\bar{e}_{i}\right\}_{i=1}^{m}$ is a set of orthogonal idempotents of $\bar{R}$. Since $N$ is a nil ideal of $R$, there are orthogonal idempotents $e_{i}$ of $R$ with $e_{i} \in \bar{e}_{i}$ such 
that $e=e_{1}+\ldots+e_{m}$ is the identity of $R$ and

$$
R=e_{1} R+\ldots+e_{m} R,
$$

where each $e_{i} R$ is an indecomposable right $R$-module. Then by (P), every $e_{i} R$ is injective or it contains a finitely generated essential socle. Suppose for example that $e_{1} R$ is injective. If $e_{1} R \cap N=0, e_{1} R$ is a minimal right ideal of $R$. For the case $e_{1} R \cap N \neq 0$, let $x$ be a non-zero element in $e_{1} R \cap N$. Since $x R$ can not contain non-zero injective submodules, $x R$ contains a finitely generated essential socle by $(\mathrm{P})$. In particular, $e_{1} R$ contains a minimal submodule $M$. Then the injective hull of $M$ in $e_{1} R$ must coincide with $e_{1} R$. Hence $\operatorname{Soc}\left(e_{1} R\right)=M$. On the other hand we have, by (3),

$$
\operatorname{Soc}\left(R_{R}\right)=\operatorname{Soc}\left(e_{1} R\right)+\ldots+\operatorname{Soc}\left(e_{m} R\right) .
$$

Combining these facts we get that $\operatorname{Soc}\left(R_{R}\right)$ is finitely generated and essential in $R_{R}$. From this and Lemma 2.1, every homomorphic image of $R$ has also this property. Then Lemma 2.4 shows that $R$ is an RM ring. Since $\operatorname{Soc}\left(R_{R}\right) \neq 0, R$ is right artinian.

The proof of Theorem 1.1 is now complete.

Remarks. A ring $R$ is called right $P C I$ if every proper (i.e. not isomorphic to $R_{R}$ ) cyclic right $R$-module is injective (cf. [7, p. 363]). As we have mentioned in the introduction, the right $\mathrm{PCI}$ rings have drawn the attention of many authors. It is remarkable to mention that every right PCI ring is right noetherian, see [4]. Now, similarly we could call a ring $R$ right PCIA if every proper cyclic right $R$-module is a direct sum of an injective module and an artinian module. Then, in the connection with Theorem 1.1, it would be worth while to test for example whether or not a right PCIA ring is right noetherian. If it were the case then the problem raised by Camillo and Krause in [22, Open problems] could be answered positively.

In [2, Theorem 3.1], Chatters characterized right noetherian rings as those rings whose cyclic right modules are direct sums of a projective module and a noetherian module. Concerning this we consider the ring $\mathbb{Z}$ of all integers. It is clear that every cyclic $\mathbb{Z}$-module is either projective or finite. Thus, the property that every cyclic right module is a direct sum of a projective module and a module with finite length cannot characterize the class of right artinian rings.

4. Rings whose ideals are right artinian rings. A ring $R$ is called hereditarily artinian if every ideal of $R$ is a right artinian ring. Every semisimple ring is hereditarily artinian, however the converse is not true in general. The structure of hereditarily artinian rings was investigated in [11], [19], [20] and recently in [6]. Using Theorem 1.1, we now improve a result given in [6, Theorem 1].

THEOREM 4.1. For a ring $R$ the following conditions are equivalent:

(a) $R$ is hereditarily artinian;

(b) $R$ and each prime ideal of $R$ are right artinian rings;

(c) $R=S \oplus F$, where $S$ is semisimple and $F$ is finite;

(d) $R$ and the prime radical $N$ of $R$ are right artinian rings; 
(e) $R$ is right artinian and $N / N^{2}$ is finite;

(f) every cyclic right $R$-module is a direct sum of an injective module whose factor modules contain no non-zero finite submodules and a finite module;

(g) every cyclic right $R$-module is a direct sum of an injective module and a finite module;

(h) every cyclic right $R$-module is a direct sum of an injective module and a module with a finite essential submodule.

Proof. (a) $\Rightarrow(\mathrm{b}) \Rightarrow(\mathrm{c}) \Rightarrow(\mathrm{d}) \Rightarrow(\mathrm{e}) \Rightarrow(\mathrm{f})$ are proved in [6, Theorem 1]; (f) $\Rightarrow(\mathrm{g}) \Rightarrow$ (h) are evident. Assume now that $R$ satisfies (h). Then $R$ is right artinian by Theorem 1.1. Let $F$ be the largest finite ideal of $R$. Then $R / F$ is a right artinian ring without non-zero finite right ideals, (cf. [5, Theorem 2]). By a similar argument to Lemma 2.1, $R / F$ satisfies (h) too. Hence every principal right ideal of $R / F$ is injective over $R / F$; therefore $R / F$ is semisimple. Thus (a) holds.

The proof of Theorem 4.1 is now complete.

An element $c$ of a ring $R$ is called regular if $c$ is not a right or left zero-divisor of $R$. Let $R \subseteq Q$ be rings. Then $R$ is defined to be a right order in $Q$ if every regular element of $R$ is a unit in $Q$ and every element $q$ of $Q$ has the form $q=a c^{-1}$ with $a, c \in R$ and $c$ regular. In this case one says also that $Q$ is the classical right quotient ring of $R$. Using Theorem 4.1(c) and some well-known facts about orders in artinian rings, we prove the following proposition.

Proposition 4.2. A ring $R$ is a right order in a hereditarily artinian ring if and only if $R$ is a direct sum of a semiprime right Goldie ring and a finite ring.

Proof. Let $R$ be a right order in a hereditarily artinian ring $Q$, and let $F$ be the largest finite ideal of $Q$. Then, by Theorem 4.1, we have

$$
Q=Q_{1} \oplus F,
$$

where $Q_{1}$ is a semisimple ring. Now, $F=(F \cap R) Q$. Since $F \cap R$ is finite, $c(F \cap R)=$ $(F \cap R) c=F \cap R$ for all regular elements $c$ of $R$; therefore $c^{-1}(F \cap R)=(F \cap R) c^{-1}=$ $F \cap R$. Hence $F=(F \cap R) Q=Q(F \cap R)=F \cap R \subseteq R$. In particular, $F$ is an ideal of $R$ containing an identity by (1). It follows that $R=S \oplus F$. Comparing with (1), we get that $S$ is a right order in $Q_{1}$. Hence $S$ is a semiprime right Goldie ring.

The converse is clear.

Remarks. In Proposition 4.2 one can prove more: if $R$ is a right order in a hereditarily artinian ring then $R$ is a direct sum of a hereditarily artinian ring and a semiprime right Goldie ring with a zero socle.

In [5, Theorem 4(a)] it was proved that the largest finite ideal of a right and left artinian ring $R$ is a direct summand of $R$. From this we can, by the same as above, show that if $R$ is a right order in a right and left artinian ring then $R$ contains a largest finite ideal which is a direct summand of $R$. Now let $R$ be a right and left order in a right and left artinian ring. Then we can show that $R_{R}$ contains a largest artinian submodule $A$ and 
${ }_{R} R$ contains a largest artinian submodule $B$. In general $A \neq B$ and they are not direct summands of $R$. This shows, for example, in the matrix ring

$$
R=\left[\begin{array}{ll}
\mathbb{Z} & \mathbb{Q} \\
0 & \mathbb{Q}
\end{array}\right],
$$

where $\mathbb{Z}$ is the ring of integers and $\mathbb{Q}$ is the field of rational numbers. We do not know whether in case $A=B, A$ is a direct summand of $R$. Assume in addition that $R$ is right and left noetherian, then $A(=B)$ is a direct summand of $R$ (see [8]).

As is well known, for a ring $R$ with the classical right quotient ring $Q, Q$ is semisimple if and only if every right $Q$-module is injective over $R$ (cf. [17, p. 58]). For the hereditarily artinian case we can prove the following result.

PROPOSITION 4.3. For a ring $R$ with the classical right quotient ring $Q$ the following conditions are equivalent:

(i) $Q$ is hereditarily artinian;

(ii) every cyclic right $Q$-module is a direct sum of an injective right $R$-module and a finite right $R$-module.

Proof. (i) $\Rightarrow$ (ii). By Theorem 4.1 and Proposition 4.2, we have

$$
Q=Q_{1} \oplus E, \quad R=R_{1} \oplus E,
$$

where $E$ is finite, $Q_{1}$ is semisimple and $R_{1}$ is a right order in $Q_{1}$. Let $M$ be a cyclic right $Q$-module. Then $M_{Q}=M Q_{1}+M E$. Clearly, $M E$ is finite. By [17, Proposition 3.8, p. 58], $M Q_{1}$ is injective over $R_{1}$, and so also over $R$, which proves (ii).

(ii) $\Rightarrow$ (i). Let $M$ be a cyclic right $Q$-module. By (ii),

$$
M_{R}=I+F,
$$

where $I_{R}$ is injective and $F_{R}$ is finite. Let $x \in I$. Then for each regular element $c$ of $R$, $x c^{-1} \in M$. By (1), $x c^{-1}=x_{1}+x_{2}\left(x_{1} \in I, x_{2} \in F\right)$; hence $x=\left(x c^{-1}\right) c=x_{1} c+x_{2} c$; therefore $x-x_{1} c=x_{2} c \in I \cap F=0$, i.e. $x c^{-1}=x_{1} \in I$. This shows that $I$ is a right $Q$-module. Similarly, $F$ is a right $Q$-module too. Now, let $\varphi$ be a $Q$-homomorphism of a right ideal $H$ of $Q$ into $I_{Q}$. Then $\varphi$ can be considered as an $R$-homomorphism of $H_{R}$ into $I_{R}$. Since $I_{R}$ is injective, there is an $R$-homomorphism $\psi$ of $Q_{R}$ into $I_{R}$ such that $\varphi=\psi \tau$, where $\tau$ is the inclusion map of $H$ into $Q$. Let $x$ and $q$ be elements of $Q, q=a c^{-1}, a, c \in R, c$ regular. Then $\psi(x q) c=\psi\left(x a c^{-1}\right) c=\psi(x a)=\psi(x) a$; therefore $\psi(x q)=\psi(x) a c^{-1}=\psi(x) q$. This shows that $\psi$ is a $Q$-homomorphism of $Q$ into $I_{Q}$, hence $I_{Q}$ is injective. By Theorem 4.1, $Q$ is then hereditarily artinian. This completes the proof of Proposition 4.3.

\section{REFERENCES}

1. E. P. Armendariz and K. E. Hummel, Restricted semiprimary rings, Ring Theory (Proc. Conf., Park City, Utah, 1971), Ed. R. Gordon (Academic Press, 1972), 1-8. 
2. A. W. Chatters, A characterisation of right noetherian rings, Quart. J. Math. Oxford Ser. (2) 33 (1982), 65-69.

3. J. Cozzens and C. Faith, Simple noetherian rings (Cambridge University Press, 1975).

4. R. Damiano, A right PCI ring is right noetherian, Proc. Amer. Math. Soc. 77 (1979), 11-14.

5. Dinh van Huynh, A note on artinian rings, Arch. Math. (Basel) 33 (1979), 546-553.

6. Dinh van Huynh, Some characterizations of hereditarily artinian rings, Glasgow Math. J. 28 (1986), 21-23.

7. C. Faith, Algebra: rings, modules and categories I (Springer, 1973).

8. S. M. Ginn and P. M. Moss, A decomposition theorem for noetherian orders in artinian rings, Bull. London Math. Soc. 9 (1977), 177-181.

9. J. S. Golan and Z. Papp, Cocritically nice rings and Boyle's conjecture, Comm. Algebra 8 (1980), 1775-1798.

10. F. Kasch, Moduln und Ringe (B. G. Teubner, 1977).

11. A. Kertész and A. Widiger, Artinsche ringe mit artinschem Radikal, J. Reine Angew. Math. 242 (1970), 8-15.

12. J. Lambek, Rings and modules (Blaisdell, 1966).

13. G. O. Michler and O. E. Villamayor, On rings whose simple modules are injective, $J$. Algebra 25 (1972), 185-201.

14. B. L. Osofsky, Rings all of whose finitely generated modules are injective, Pacific J. Math. 14 (1964), 645-650.

15. P. F. Smith, Some rings which are characterised by their finitely generated modules, Quart. J. Math. Oxford Ser. (2) 29 (1978), 101-109.

16. P. F. Smith, Rings characterized by their cyclic modules, Canad. J. Math. 31 (1979), 93-111.

17. B. Stenström, Rings of quotients (Springer, 1975).

18. P. Vámos, The dual of the notion of "finitely generated", J. London Math. Soc. (2) 43 (1968), 643-646.

19. A. Widiger, Lattice of radicals for hereditarily artinian rings, Math. Nachr. 84 (1978), 301-309.

20. A. Widiger and R. Wiegandt, Theory of radicals for hereditarily artinian rings, Acta Sci. Math. (Szeged) 39 (1977), 303-312.

21. R. Yue Chi Ming, On flatness, $p$-injectivity and von Neumann regularity, Bull. Soc. Math. Belg. Sér. B 35 (1983), 97-109.

22. Rings, modules and radicals (Colloq., Keszthely, 1971), Ed. A. Kertész, Colloq. Math. Soc. Janos Bolyai, vol. 6 (North-Holland, János Bocyai Math. Soc., 1973).

InStitute of Mathematics

P.O. Box 631 Bo Hô

HANOI-VIETNAM 